\title{
DESIGN AND MULTIPLIER-LESS REALIZATION OF MATCHED FILTERS WITH VARIABLE FRACTIONAL DELAY FOR SOFTWARE RADIO RECEIVERS
}

\author{
S. C. Chan and K. M. Tsui
}

Department of Electrical and Electronic Engineering,

The University of Hong Kong, Pokfulam Road, Hong Kong.

\begin{abstract}
This paper studies the design and multiplier-less realization of variable fractional delay matched filters (VFD-MFs), which provide matching filtering and variable fractional delay of the filter output. It offers greater flexibility and lower delay in symbol-timing adjustment than directly cascading a match filter with a fractional delayer. The design of VFD-MFs, which can be viewed as a variable digital filter (VDF) design problem subject to the matched filtering condition, is formulated as a second order cone programming (SOCP) problem with least square design criteria. The proposed VFD-MFs can be efficiently implemented using the Farrow structure. By employing sum-of-power-of-two (SOPOT) coefficients and the multiplier block (MB) technique, very efficient multiplier-less realization of the VFD-MF with low hardware complexity is obtained. A design example is given to demonstrate the effectiveness of the proposed approach.
\end{abstract}

\section{INTRODUCTION}

In a conventional linear modulation receiver, match filtering (or correlation demodulator) is performed on the received signal and its output is sampled at the end of each symbol interval. The output is then fed to a symbol timing recovery circuit to recover the symbol timing. A fractional delay digital filter (FDDF) is usually employed to interpolate the match filter output so that the demodulated signal can be sampled at the right timing instant to minimize inter-symbol interference (ISI) [1]. If the IF is implemented digitally, channelization using a cascade of band-limiting filters and sampling rate conversion might be required before feeding to the digital receiver [2]. Conventionally, the match filter and FDDF are implemented separately. More recently, there is an interest in combining these components together [3-5]. There are two potential advantages of the latter approach: 1) the overall system delay can be reduced as we shall demonstrate later in the design example, 2) the specifications of the match filter and FDDF can be jointly optimized to reduce the implementation complexities for a given specification. In [3], a polyphase filter approach is employed where a number of fractional sample delayed versions of the match filter are precalculated and stored. On the other hand, the combined match filter-interpolator in $[4,5]$ is designed using the least square approach and implemented using the Farrow structure [6]. For convenience, we shall call such filters variable fractional delay matched filters (VFD-MFs). VDFs are filters whose frequency characteristics can be varied on-line by a set control parameters. FDDFs are VDFs with variable fractional sample delay [6-8].

In this paper, the design and multiplier-less realization of VFD-MFs with lower system delay are studied. The design of VFD-MFs is considered as the design of a VDF subject to the matched filtering condition, and it is formulated as a second order cone programming (SOCP) $[9,10]$ problem with least square design criterion with peak stopband constraints. For illustrative purpose, we shall consider the design of a pair of transmit and receive filters which form a Nyquist channel. Since it is difficult to satisfy exactly the zero ISI condition [1] for all fractional delay of the MF output over which the spectral parameter is to varied, the Nyquist condition is approximated by assuming that the transmit filter and the VFD-MF constitute a raised cosine response with variable fractional sample delay. The SOCP framework is more flexible than the method in [3-5] in that additional convex quadratic inequality constraints such as peak error constraints in the stopband can be incorporated readily. Since SOCP is a convex optimization problem, the optimal solution, if it exists, is guaranteed. The proposed VFDMFs is also implemented using the Farrow structure. Since the subfilter coefficients in the Farrow structure are fixed, they can be implemented efficiently using canonical signed digits (CSD) or sum-of-power-of-two (SOPOT) coefficients [11], which can be implemented as limited number of shifters and adders, giving rise to multiplier-less realization with very low implementation complexity. A number of methods [11-14] have been proposed to determine the SOPOT approximation in such multiplier-less implementation. Here, the random search algorithm reported in [12] is employed, because of its flexibility in accommodating a wide variety of constraints. To reduce the arithmetic complexity further, the subfilters are implemented in their transposed form and realized as multiplier block (MB) [14]. Using the MB technique [15], the total number of additions can be kept to minimal by reusing the intermediate results generated. Consequently, apart from the limited number of multipliers required in the Farrow structure, the proposed VFD-MFs can be implemented without any multiplications.

The paper is organized as follows: Section II is devoted to the properties of the Nyquist filters and the zero inter-symbol interference condition. The formulation of the VFD-MFs design problem using SOCP is introduced in section III. The incorporation of peak error constraints on the VFD-MFs will also be considered. Section IV describes the multiplier-less implementation of the VFD-MF. Design examples are given in Section $V$ to demonstrate the effectiveness of the proposed approach, and finally, conclusion is drawn in Section VI.

\section{Properties of The Nyquist Filters}

In digital communication systems, it is often required that the cascade of transmit and receive filters form a Nyquist filter in order to avoid intersymbol interference (ISI) [1]. In general, Nyquist filters are lowpass filters and their desired frequency responses are given by:

$$
G_{d}(\omega)=\left\{\begin{array}{ll}
e^{-f \omega r} & 0<\omega<\omega_{p} \\
0 & \omega_{x}<\omega<\pi
\end{array},\right.
$$

where $\tau=(L-1) / 2-D$ is the group delay, $D$ is the prescribed delay reduction parameter, which is assumed to be a positive integer, over a linear-phase filter of odd length $L$, $\omega_{p}=(1-\rho) \pi / M$ and $\omega_{s}=(1+\rho) \pi / M$ are the passband and stopband cutoff frequencies respectively; $M$ is the oversampling factor and $0<\rho<1$ is the rolloff factor. On the other hand, the impulse response of a Nyquist filter satisfies the following zero ISI or zero-crossing conditions [16]:

$$
g(n)=\left\{\begin{array}{ll}
1 / M & n=\tau \\
0 & n=\tau \pm k M, k=1,2, \ldots
\end{array} .\right.
$$

For linear-phase Nyquist filter (i.e. $D=0$ ), $\tau$ is the centre of symmetry of its impulse response. Alternatively, the desired frequency response can be approximated in the frequency domain as the following raised cosine response: 


$$
G_{d}(\omega)= \begin{cases}e^{-j \omega r}, & 0<\omega<\omega_{p} \\ e^{-j \omega r} \cdot \cos ^{2}\left[\left(\omega-\omega_{p}\right) \mathrm{M} / 4 \rho\right], & \omega_{p}<\omega<\omega_{s} . \\ 0, & \omega_{s}<\omega<\pi\end{cases}
$$

\section{Problem formulation}

In a VDF [14], the desired response $H_{d}(\omega, \phi)$ is a function of a spectral parameter $\phi$. The spectral characteristics of a VDF can therefore be continuously varied by changing $\phi$. The impulse response of the VDF under consideration is assumed to be a linear combination of a polynomial basis function of the spectral parameter $\phi$ and subfilter coefficients $h_{l}(n)$, and it is given by:

$$
h(n, \phi)=\sum_{l=0}^{L-1} h_{l}(n) \cdot \phi^{\prime},
$$

where $\phi$ is assumed to vary linearly over a finite interval. The $\mathrm{z}$-transform of the polynomial-based VDF is then given by:

$$
H(z, \phi)=\sum_{l=0}^{L-1}\left[\sum_{n=0}^{N-1} h_{l}(n) z^{-n}\right] \cdot \phi^{l}=\sum_{l=0}^{L-1} H_{l}(z) \cdot \phi^{l},
$$

where $H_{l}(z)=\sum_{n=0}^{N-1} h_{l}(n) z^{-n}$ is the $l$-th FIR subfilter. (3-2) suggests a very useful structure for implementing VDF called the Farrow structure, which is shown in figure 1. To simplify the notation, substitute $m=n+N l$ and $z=e^{j \omega}$ into (3-2), one gets:

$$
H\left(e^{j \omega}, \phi\right)=\sum_{m=0}^{L N-1} a_{m} \phi^{\prime} e^{-j \omega m},
$$

where $n=\bmod (m, N)$ and $l=(m-n) / N$.

As mentioned earlier, the combination of the receive filter and the FDDF can be viewed as a VDF. More precisely, transmit filter $H_{T}(z)$ and receive VDF, $H(z, \phi)$ together approximate the raised cosine response with the following tunable fractional delay:

$$
G_{d}(\omega, \phi)=\left\{\begin{array}{lr}
e^{-j \omega \tau(\phi)}, & 0<\omega<\omega_{p} \\
e^{-j \omega \tau(\phi)} \cos ^{2}\left[\left(\omega-\omega_{p}\right) \mathrm{M} / 4 \rho\right], & \omega_{p}<\omega<\omega_{s} \\
0, & \omega_{s}<\omega<\pi
\end{array}\right.
$$

where $\tau(\phi)=\left(N+N_{T}-2\right) / 2-D+\phi$ is the group delay of the VDF; $N_{T}$ is the length of the transmit filter. Without loss of generality, the transmit filter is assumed to be a linear-phase square-root raised cosine filter. On the other hand, the overall system response $G\left(e^{j \omega}, \phi\right)$ in term of $H(z, \phi)$ is:

$$
G\left(e^{j \omega}, \phi\right)=H_{T}\left(e^{j \omega}\right) \cdot H\left(e^{j \omega}, \phi\right) .
$$

Using (3-3), (3-5) can be rewritten more compactly in matrix form of the design parameters $a$ as follows:

$$
\begin{aligned}
& G\left(e^{j \omega}, \phi\right)=\boldsymbol{a}^{T}\{\boldsymbol{c}(\omega, \phi)-j \boldsymbol{s}(\omega, \phi)\}, \\
& \boldsymbol{a}=\left[a_{0}, \ldots, a_{L N-1}\right]^{T}=\left[\boldsymbol{h}_{\boldsymbol{\theta}}{ }^{T}, \ldots, \boldsymbol{h}_{L-1}{ }^{T}\right]^{T}, \\
& \boldsymbol{h}_{l}=\left[h_{l}(0), \ldots, h_{l}(N-1)\right]^{r} \text { for } l=0,1, \ldots, L-1 \text {, } \\
& \text { where } c(\omega, \phi)=\operatorname{Re}[e l(\omega, \phi)], s(\omega, \phi)=\operatorname{Im}[e l(\omega, \phi)] \text {; } \\
& \boldsymbol{e} 1(\omega, \phi)=H_{f}\left(e^{j \omega}\right) \cdot\left[\boldsymbol{e}(\omega)^{\gamma}, \phi^{\prime} \cdot \boldsymbol{e}(\omega)^{T}, \ldots, \phi^{l-i} \cdot \boldsymbol{e}(\omega)^{7}\right]^{7} ; \\
& \boldsymbol{e}(\omega)=\left[1, e^{-j \omega} \ldots, e^{-j(\lambda-1) \omega}\right]^{T} .
\end{aligned}
$$

where $\operatorname{Re}[\bullet]$ and $\operatorname{Im}[\bullet]$ denote respectively the real and imaginary parts of the elements inside the square bracket. Here, we shall approximate the desired response $G_{d}(\omega)$ by $G(z)$ in the least-square sense. That is:

$$
\begin{aligned}
& E_{L S}=\zeta_{2} W(\omega, \phi) \cdot\left|G\left(e^{i \omega}, \phi\right)-G_{d t}(\omega, \phi)\right|^{2} d \omega d \phi, \\
& (\omega, \phi) \in \Omega
\end{aligned}
$$

where $W(\omega, \phi)$ is a positive weighting function, and $\Omega$ is the (frequency, tuning range) of interest. The advantage of minimizing $E_{L S}$ is that it can be written as a quadratic function of $a$ :

$$
\min _{\boldsymbol{a}} \delta \quad \text { subject to } \delta \geq \boldsymbol{a}^{T} \boldsymbol{Q a}-2 \boldsymbol{a}^{T} \boldsymbol{p}+k,
$$

where $Q=\int_{2} W(\omega, \phi) \cdot\left[c(\omega, \phi) c(\omega, \phi)^{T}+s(\omega, \phi) s(\omega, \phi)^{T}\right] d \omega d \phi$, $\boldsymbol{p}=\int_{h} W(\omega, \phi)\left\{c(\omega, \phi) \operatorname{Re}\left[G_{d}(\omega, \phi)\right]+s(\omega, \phi) \operatorname{Im}\left[G_{d}(\omega, \phi)\right]\right\} d \omega d \phi$ and $k=\int_{\Omega} W(\omega, \phi)\left|G_{d}(\omega, \phi)\right|^{2} d \omega d \phi$. The optimal LS solution is given by $a_{L S}=Q^{-1} p$. Next, we shall formulate these problems as a convex programming problem and solved using second order cone programming (SOCP) [9-10]. The advantage of formulating the objective function as a convex problem such as SOCP or semidefinite programming (SDP) is that the resulting problem is convex and the optimal solution, if it exists, can be found. In addition, other linear equalities or convex quadratic constraints can easily be incorporated, as we shall illustrate later in this section. By defining $\overline{\boldsymbol{Q}}=\left[\begin{array}{ll}\boldsymbol{O}_{N} & \boldsymbol{Q}^{1 / 2}\end{array}\right], \overline{\boldsymbol{p}}=\boldsymbol{Q}^{-1 / 2} \boldsymbol{p}$, $\bar{k}=\boldsymbol{p}^{T} \boldsymbol{Q}^{-1} \boldsymbol{p}-k, \boldsymbol{x}=\left[\begin{array}{ll}\delta & \boldsymbol{a}^{T}\end{array}\right]^{T}$ and $\boldsymbol{c}=[1,0, \ldots .0]^{T}$, one can reformulate (3-8) as the following SOCP:

$$
\min _{x} c^{T} x \quad \text { subject to } c^{T} x-\bar{k} \geq\|\bar{Q} x-\bar{p}\|_{2} .
$$

where $\boldsymbol{O}_{N}$ is a $N$ row zero vector.

To avoid excessive sidelobe of the LS solution, additional peak constraints are imposed on the stopband. Let $\varepsilon_{p}$ be the peak ripple to be imposed in a frequency band $\omega \in\left[\omega_{1}, \omega_{2}\right]$ (a collection of frequency bands is also feasible). Then, the peak error constraint can be written as:

$$
\left|H\left(e^{j \omega}, \phi\right)\right| \leq \varepsilon_{p}, \omega \in\left[\omega_{1}, \omega_{2}\right],(\omega, \phi) \in \Omega .
$$

Digitizing the frequency variable $\omega$ and control parameter $\phi$ over a dense set of frequencies $\left\{\omega_{i}, 1 \leq i \leq K_{1}\right\}$ and control parameters $\left\{\phi_{k}, 1 \leq k \leq K_{2}\right\}$ in the range of interests, the constraints in (3-10) becomes:

$$
\begin{aligned}
& \varepsilon_{r}- {\left[\alpha_{R}^{2}\left(\omega_{i}, \phi_{k}\right)+\alpha_{t}^{2}\left(\omega_{i}, \phi_{k}\right)\right]^{1 / 2} \geq 0, } \\
& \alpha_{k}\left(\omega_{i}, \phi_{k}\right)=\left|\boldsymbol{a}^{r} c 1\left(\omega_{i}, \phi_{k}\right)\right|, \alpha,\left(\omega_{i}, \phi_{k}\right)=\left|\boldsymbol{a}^{r} s 1\left(\omega_{i}, \phi_{k}\right)\right| ;
\end{aligned}
$$

where $\boldsymbol{c} 1\left(\omega_{i}, \phi_{k}\right)=\operatorname{Re}\left[e 2\left(\omega_{i}, \phi_{k}\right)\right], \boldsymbol{s} 1\left(\omega_{i}, \phi_{k}\right)=\operatorname{Im}\left[e 2\left(\omega_{i}, \phi_{k}\right)\right]$;

$$
\boldsymbol{e} 2\left(\omega_{i}, \phi_{k}\right)=\left[\boldsymbol{e}\left(\omega_{i}\right)^{T}, \phi_{k}^{\prime} \cdot \boldsymbol{e}\left(\omega_{i}\right)^{T}, \ldots, \phi_{k}^{i-1} \cdot e\left(\omega_{i}\right)^{T}\right]^{T} .
$$

Or equivalently

$$
\varepsilon_{p} \geq\left\|\boldsymbol{R}_{i . h} x\right\|_{2}
$$

where $\quad \boldsymbol{R}_{i, k}=\left[\begin{array}{cc}0 & c 1\left(\omega_{i}, \phi_{k}\right)^{T} \\ 0 & s 1\left(\omega_{i}, \phi_{k}\right)^{r}\end{array}\right]$.

The resulting constraints on the peak ripples can then be augmented to the existing constraints in (3-9).

\section{MUltiplier-LESS REALIZATION}

In this section, the multiplier-less realization of the proposed MF-VFD is described. As mentioned earlier, the VDF is implemented as a Farrow structure with the subfilter coefficients being represented as SOPOT coefficients [11]. To further reduce the implementation complexity, the multiplierblock (MB) technique is also used. The basic idea of MB is to reduce the redundancies found in implementing all SOPOT coefficients by removing any possible common subexpressions in their representations. More precisely, the coefficients $h_{l}(n)$ in the $l$-th subfilter $H_{l}(z)$ are represented in the following SOPOT form: 


$$
\hat{h}_{f}(n)=\sum_{r=1}^{R} u_{i, n, r} 2^{a_{r}},
$$

with $u_{l, n, r} \in\{-1,1\}$ and $a_{r} \in\{-g, \ldots,-1,0,1, \ldots, g\}$, where $g$ is a positive integer and its value determines the range of the coefficients, and $R$ is the number of terms used in the coefficient approximation. The coefficient multiplication can then be implemented as limited number of shifts and additions, giving rise to multiplier-less realization. These SOPOT coefficients can be obtained by a number of methods [11-14]. Here, we shall employ the random search algorithm reported in [12]. One advantage of this algorithm is that different types of constraints can be easily incorporated. In particular, the constraints for the peak passband error, peak stopband error, peak group delay error and peak distortion error are incorporated in our design. To determine the SOPOT coefficients, the following objective function is minimized for $\phi \in[-0.5,0.5]$ :

$$
\begin{aligned}
& \min \left\langle T_{\text {SOPOOT }}\right\rangle \text { subject to }\left\{\begin{array}{l}
\delta_{p} \leq \delta_{p-\max } \\
\delta_{s} \leq \delta_{s-\max } \\
\delta_{d} \leq \delta_{d-\max } \\
\delta_{i} \leq \delta_{i-\max }
\end{array}\right. \\
& \text { where } \delta_{p}=\max _{0<|\omega|<\omega p}\left\langle\left|H_{d}\left(e^{j \omega}, \phi\right)-\hat{H}\left(e^{j \omega}, \phi\right)\right|\right\rangle
\end{aligned}
$$$$
\text { is the peak passband error, }
$$

$$
\delta_{s}=\max _{\omega_{s}\{\langle\omega|<\pi}\left\langle\left|H_{d}\left(e^{j \omega}, \phi\right)-\hat{H}\left(e^{j \omega}, \phi\right)\right|\right\rangle
$$

is the peak stopband error,

$$
\delta_{d}=\max _{\hat{\alpha}\left\{\left\{|\omega| \leqslant \omega_{p}\right.\right.}\langle|\tau(\phi)-\hat{\tau}(\phi)|\rangle
$$

is the peak group delay error, and

$$
\delta_{i}=\max \left\langle\frac{\sum_{k=1}^{L 1}|\hat{g}(\tau-k M)|+\sum_{k=1}^{L 2}|\hat{g}(\tau+k M)|}{|\hat{g}(\tau)|}\right\rangle
$$

is the peak distortion error, which is used to measure the ISI error; $L 1=\lfloor\tau / M\rfloor, L 2=\lfloor(L-1-\tau) / M\rfloor$ and $\lfloor\alpha\rfloor$ denotes the integer just less than or equal to $\alpha . T_{\text {SOPOT }}$ is the total number of SOPOT terms used to implement all the SOPOT coefficients. $\hat{H}\left(e^{j \omega}, \phi\right)$ and $\hat{\tau}(\phi)$ are respectively the frequency and group delay responses of the candidate multiplier-less MF-VFD. $\hat{g}(n)$ is the impulse response obtained by cascading the candidate multiplier-less MF-VFD and the transmit filter with different fractional sample delays. $\delta_{p-\max }, \delta_{s-\max }, \delta_{d-\max }$ and $\delta_{i-\max }$ are the maximum tolerance of the passband, stopband, group delay and distortion errors, respectively. It should be noted that other meaningful measures, say other performance measure of the match filter or power consumption, can also be used instead. In the random search algorithm, the real-valued coefficients are first obtained as described in section III. Let $\boldsymbol{b}$ be the vector containing these real-valued coefficients. Then, the algorithm repetitively calculates a candidate SOPOT vector $\boldsymbol{b}_{\mathrm{c}}$, which is given by:

$$
\boldsymbol{b}_{c}=\left\lfloor\boldsymbol{b}+\lambda \boldsymbol{b}_{p}\right\rfloor_{\text {SOPOT }},
$$

where $b_{p}$ is a random vector with elements chosen in the range $\pm 1, \lambda$ is a user-defined variable used to control the size of the neighborhood to be searched, and $[\cdot]_{\text {SOPOT }}$ is the rounding operator that converts every element inside the input vector to its closest SOPOT value for a given value of $g$. The performance measures $\delta_{p}, \delta_{s}, \delta_{d}$ and $\delta_{i}$ of the new SOPOT coefficients are then calculated. The set that yields the minimum number of terms for implementing the SOPOT coefficient while satisfying the given specifications is declared as the solution. Since this is a random search algorithm, the longer the searching time, the higher the chance of finding the optimal solution. To implement this multiplier-less VFD-MF using MB, let's consider its implementation in figure 2 . It can be seen that each subfilter is implemented in their transposed form, where the input signal $x(n)$ is multiplied with a large number of constant coefficients in SOPOT form. The redundant additions in these SOPOT products can be reduced using a MB, which greatly reduces the arithmetic complexity. Interested readers are referred to [15] for more details. We now present a design example.

\section{Design EXAMPLes}

In this example, variable fractional delay match filters with low system delay are designed using the proposed approach. The design problem was solved by the SeDuMi Matlab Toolbox [17] on a Pentium 4 PC. The specifications are: $N=17, L=5, M=4, \rho=0.5$ and $\phi \in[-0.5,0.5]$. The delay reduction parameter $D$ is chosen to be 2 so that the group delay of the proposed MF-VFD ranges from 5.5 to 6.5. The transmit filter is assumed to be a linear-phase square-root raised cosine filter of length $N_{T}=17$. The peak error constraint is imposed to limit the sidelode in the stopband to $20 \mathrm{~dB}$ with $K_{1}=50$ and $K_{2}=50$. The passband, stopband, group delay and ISI error over the entire range of the tuning parameter $\phi$ are plotted as dash-dotted line in figure 2. It can be seen that the corresponding worst-case passband, stopband, group delay and ISI errors are respectively $0.003499,0.1,0.02643$ and 0.0151 . The computational time is about 45.828 seconds.

As mentioned earlier, the subfilter coefficients, which are fixed, can be implemented using SOPOT coefficients. These SOPOT coefficients are determined as described in section IV. As an illustration, the passband specification is relaxed so that a tighter group delay specification can be achieved. The target specifications of the proposed multiplier-less MF-VFD are: $\delta_{p-\text { max }}=0.00577 \quad(0.05 \mathrm{~dB}), \quad \delta_{s-\max }=0.106 \quad(19.5 \mathrm{~dB})$, $\delta_{d-\max }=0.02$ and $\delta_{i-\max }=0.015$. It can be seen from the solid line in figure 2 that the worst-case group delay is improved at the expense of slightly increase in passband error. This substantiates the usefulness of the proposed multiplier-less realization method. It should be noted that the total number of multipliers required to implement all the real-valued coefficients of the MF-VFD is 86 . On the other hand, the realization using SOPOT coefficients requires only 202 adders. After using the MB technique, the number of adders is further reduced to 74 , which is about $36.6 \%$ of the hardware resources required for directly implementing all the SOPOT coefficients. The frequency and group delay responses of the proposed multiplier-less MF-VFD are shown in figures $3 a$ and $3 b$ respectively. Table 1 summarizes the design results in this example.

\section{Conclusion}

The design of variable fractional delay matched filters (VFD-MFs) with lower system delay using second order cone programming and its multiplier-less implementation have been represented. It is implemented as a Farrow structure with the subfilter coefficients being SOPOT coefficients and implemented as MB. A design example is given to demonstrate the effectiveness of the proposed approach. 


\section{REFERENCES}

[1] J. G. Proakis and M. Salehi, "Contemporary communication systems using Matlab," Brooks/Cole, Thomson Learning, 2000.

[2] S. C. Chan and K. S. Yeung, "On the application of variable digital filters (VDF) to the realization of software radio receivers," in Proc. ISCAS'2003, vol. 3, pp. 562-565, May 2003.

[3] L. P. Sabel, A. Yardim, G. D. Cain and T. I. Laakso, "Improved delay root-Nyquist filters for symbol synchronization in PCS receivers," in Proc. IEEE Global Communications Conference, pp. 1302-1324, Nov. 1995.

[4] Jussi Vesma, Tapio Saramaki and Marku Renfors, "Combined matched filter and polynomial-based interpolator for symbol synchronization in digital receivers," in Proc. ISCAS'1999, vol. 3, pp. 94-97, May 1999

[5] M. Henker and G. Fettweis. "Combined Filter for Sample Rate Conversion, Matched Filtering, and Symbol Synchronization in Software Radio Terminals," in Proc. European Wireless 2000, pp. 61-66, September 2000.

[6] C. W. Farrow, "A Continuously Variable Digital Delay Element," in Proc. ISCAS, vol. 3, pp. 2641-2645, 1988.

[7] J. Vesma and T. Saramaki, "Design and properties of polynomial-based fractional delay filters," in Proc. of ISCAS 2000, pp.104-107.

[8] T. I. Laakso, V. Valimaki, M. Karjalainen and U. K. Laine, "Splitting the unit delay, tools for fractional delay filter design," IEEE Signal Processing Mag., pp.30-60, Jan. 1996.

[9] M. S. Lobo, L. Vandenberghe, S. Boye and H. Lebret, "Applications of second-order cone programming", Linear Algerba Application, vol. 248, pp. 193-228, Nov. 1998.

[10] J. O. Coleman and D. P. Scholnik, "Design of non-linear phase FIR filters with second-order cone programming", in Proc. MWSCAS 1999, vol. 1, pp. 409-412, Aug. 1999.

[11] Y. C. Lim and S. R. Parker, "FIR filter design over a discrete power-of-two coefficient space," IEEE Trans. ASSP-31, pp. 583-591, April 1983.

[12] C. K. S. Pun, Y. C. Wu, S. C. Chan and K. L. Ho, "An efficient design of fractional-delay digital FIR filter using Farrow structure," Proceedings of the $11^{\text {th }}$ IEEE Signal Processing Workshop on Statistical Signal Processing, pp. 595-598, 2001.

[13] G. Wade, A. Roberts and G. Williams, "Multiplier-less FIR filter design using a genetic algorithm," IEEE Vision, Image and Signal Processing, vol. 141, no. 3, pp. 175-180, June 1994.

[14] K. S. Pun, S. C. Chan, K. S. Yeung and K. L. Ho, "On the Design of FIR and IIR Digital Filters with Variable Frequency Characteristics," IEEE Trans. Circuits Syst. II, vol. 49, no. 11, pp. 689-703, Nov, 2002.

[15] A. G. Dempster and M. D. MacLeod, "Use of minimumadder multiplier blocks in FIR digital filters," IEEE Trans. Circuits Syst. II, pp. 569-577, Sept. 1995.

[16] Y. Wisutmethangoon and T. Q. Nguyen, "A Method for Design of $M$ th-Band Filters," IEEE Trans. Signal Processing, vol. 47, no. 6, pp. 1669-1678, June, 1999.

[17] J. F. Sturm, "Using SeDuMi 1.02, a MATLAB toolbox for optimization over symmetric cones," Optim. Meth. Softw., vol. 11-12, pp. 625-653, 1999.

\begin{tabular}{|c|c|c|}
\hline Fin & Real-valued & SOPOT \\
\hline 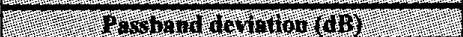 & 0.0303 & 0.0450 \\
\hline 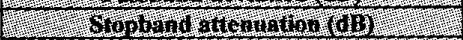 & 20 & 19.7 \\
\hline T Group defy wor (crandes) & 0.0265 & 0.0177 \\
\hline 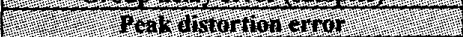 & 0.0151 & 0.0150 \\
\hline 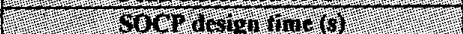 & 45.828 & $\mathrm{~N} / \mathrm{A}$ \\
\hline 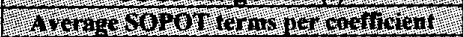 & N/A & 3.61 \\
\hline Withr required for sovot before WD & N/A & 202 \\
\hline Aderes requind for SOPOT after Mi & $\mathrm{N} / \mathrm{A}$ & 74 \\
\hline
\end{tabular}

Table 1. Design results of the variable fractional delay match filters.

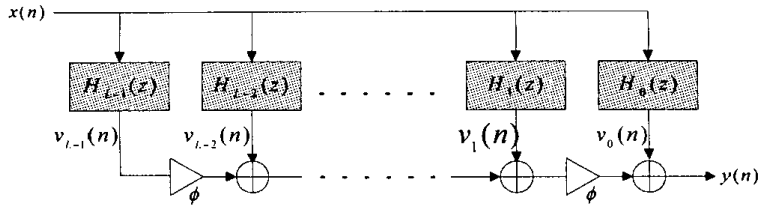

1a)



1b)

Figure 1: Implementation of the variable digital filter (VDF) (a) Farrow structure (b) transposed form of sub-filters.

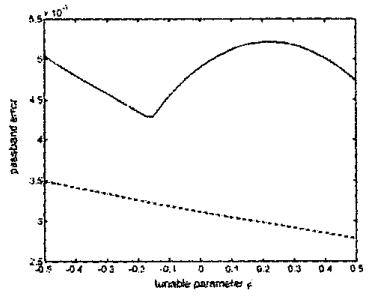

2a)

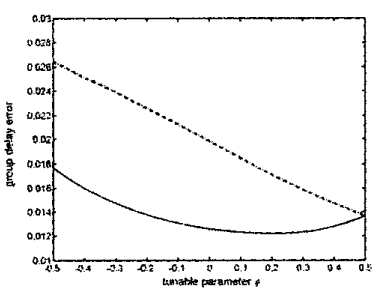

2c)



2b)

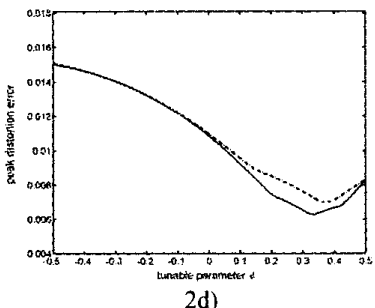

2d)
Figure 2: a) Passband errors, b) stopband errors, c) group delay errors and d) peak distortion errors of the VFD-DFs with real-valued (dash-dotted line) and SOPOT (solid line) coefficients.



3a)

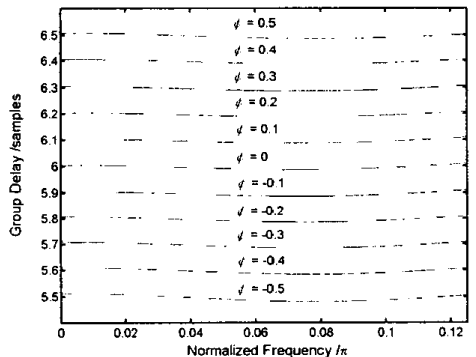

3b)

Figure 3: a) Frequency response and b) group delay response of the multiplier-less VFD-MF with evenly sampled tunable parameter $\phi$. 